\title{
Study on the Improvement of Flexibility for an Industrial Robot Based on Machine Vision
}

\author{
Jiwu Wang ${ }^{1}$ \\ School of Mechanical, Electronic and Control Engineering, Beijing Jiaotong University \\ Beijing 100044, China ${ }^{1}$ \\ Xianwen Zhang', Weining Zhang \\ School of Mechanical, Electronic and Control Engineering, Beijing Jiaotong University \\ Beijing 100044, China ${ }^{1}$ \\ Sugisaka Masanori ${ }^{2}$ \\ ALife Robotics Corporation Ltd.,Oaza Shimohanda, Oita 870-1112, Japan² \\ E-mail: jwwang@bjtu.edu.cn; ms@alife-robotics.co.jp
}

\begin{abstract}
In order to improve the applications for an industrial robot, it is necessary to increase its flexibility and accuracy. The machine vision technology is an effective solution. In order to reduce the influence of the size, deformation etc., the target extraction method with fusion of color space and moment invariants is developed. It makes the multiple targets extracted accurately and robustly. The experiments results showed that the developed algorithms are robust, and the robot's flexibility can be improved.
\end{abstract}

Keywords: Arm robot, Target recognition, Flexible control, Machine vision

\section{Introduction}

With the development of new technology and the higher requirements of product quality and production efficiency, more and more production lines are used in modern companies. ${ }^{1}$ But skillful workers with experience can not be trained in a short time and its number is not enough. Many automation machines are required to operate like skillful workers. Compared with an operator, the robot rarely makes mistakes if its working conditions are not changed. And its efficiency and reliability is higher than an operator. Especially in the danger or high working intensity environment, it is more appropriate than human beings. Then various robots are developed and applied in the most advanced company.

Generally, most robots firstly can only be used for some simple, repetitive and single jobs. But now the further applications are required to be developed. On the other hand, the repetitive positioning accuracy can not be satisfied if an arm robot is used for a longer time. Although some feedback or sensor fusion technology is adopted, the effective solutions are not enough.

With the development of the CCD camera technology, machine vision gets more attention. The CCD camera not only works like human being eyes, but also can provide more information than other sensors. With the pattern recognition algorithms, the CCD camera can 
provide multiple target positions in a captured image. ${ }^{2}$ Moreover, it can calculate the end position of the arm robot in a captured image if necessary. In a word, with a CCD camera, the operating flexibility and repetitive positioning accuracy can all be improved.

In the application of machine vision, in order to get robust and reliable image processing results, the lighting conditions and image processing efficiency should be considered carefully. In this paper, considering the practical application requirements, the fusion technique of true color space and moment invariant is applied in the pattern recognition.

The structure of the arm robot is shown as figure 1. A is the CCD camera, B is the control box, $\mathrm{C}$ is a step motor to control the upper arm, D is a step motor to control the forearm, $\mathrm{E}$ is a step motor to control the hand, $\mathrm{F}$ is a support just like the shoulder, $\mathrm{G}$ is the upper arm, $\mathrm{H}$ is the forearm. The length of the upper arm and forearm is $400 \mathrm{~mm}$ respectively. The height between the camera and ground is $700 \mathrm{~mm}$.

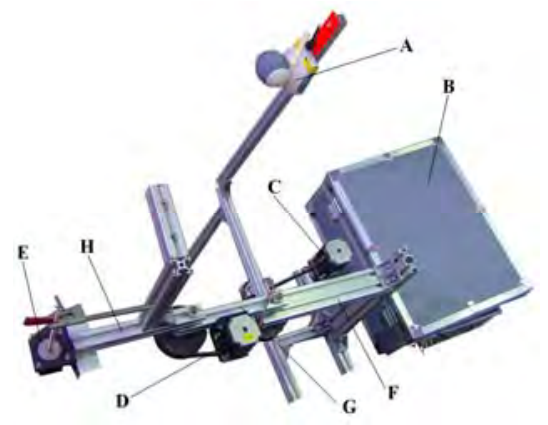

Fig. 1 The structure illustration of the arm robot

The control relationship among all hardware is illustrated in figure 2. The CCD camera is used to determine the target position. Three motors $(\mathrm{C}, \mathrm{D}, \mathrm{E})$ is controlled by the PC through motion control card. The working process is as following: 1) After some targets are detected by the CCD camera, they will be extracted by the image processing algorithms in PC(An industrial computer), and their positions will be calculated. 2) Based on the determined target positions, the computer will calculate rotation angles for each joint, and three motors will run together to make the hand reach each target.

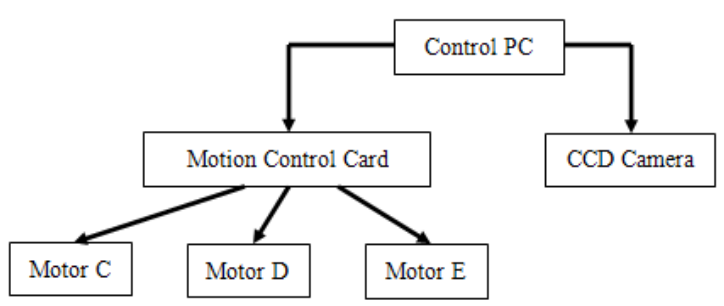

Fig. 2 The control relationship about all hardware

The developed software system is shown in figure 3. In the centre part, it is the target display area. The right part is the motor control area.

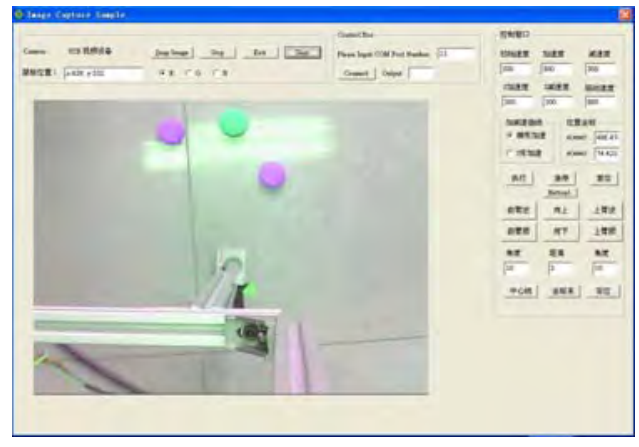

Fig. 3 Illustration of the developed software interface

\section{The Target Extraction Method}

In order to extract targets from the captured image at a higher speed, the color based target detection method is developed. At the same time, to improve recognition ratio, the moment invariant algorithms are then applied to determine whether the separated objects are targets or not.

In normal case, the triple components $(\mathrm{R}, \mathrm{G}, \mathrm{B})$ of each pixel can be used to extract the target area in a common RGB image. But it is more difficult to determine an appropriate color threshold suitable for different lighting conditions, which will bring unstable for the following target recognition.

In order to reduce the influence of different lighting conditions, a color model in the chromatic color space is built. Chromatic colors, also known as pure colors in the absence of luminance, are defined by a normalization of the triple components (R, G, B). The process to build target color model is as follows: 
1) Capture some common RGB color images of the target under different lighting conditions, and take them as sample images. Then extract the typical RGB target color areas manually from these sample images as sample colors.

2) Transform these sample colors from the RGB color space to the chromatic color space by a normalization process. Here color green is redundant because of $r+g+b=1$.

$$
\begin{aligned}
& r=R /(R+G+B) \\
& b=B /(R+G+B)
\end{aligned}
$$

3) Calculate the mean value and covariance of sample colors in the chromatic color space.

$$
\begin{aligned}
& \bar{x}=\frac{\sum_{n} x}{n} \\
& C=\frac{\sum_{n}(x-\bar{x})(x-\bar{x})^{T}}{n-1}
\end{aligned}
$$

Where, $\mathrm{x}$ is a pair of normalization components $(\mathrm{r}, \mathrm{b})$ for each pixel in the chromatic color space; $\bar{x}$ is the mean value and $C$ is the corresponding covariance.

4) The possibility of the target color for each pixel in the detected image can be calculated as follows:

$$
P_{x}=\exp \left[-0.5(x-\bar{x}) C^{-1}(x-\bar{x})\right]
$$

When the color model is used to extract target, we should firstly transform each pixel from the RGB color representation to the chromatic representation. Then a pair of chromatic color values for each pixel is input the color model to decide whether that pixel has the same color as the target. By this way, the whole image will be separated by the target areas and non-target areas.

The next step is to extract the targets with moment invariants in the above separated image based on the color model. The geometric moment invariants are calculated by the following formula ${ }^{3,4}$ :

$$
\mathrm{M}_{\mathrm{pq}}=\iint_{\mathrm{D}} \mathrm{x}^{\mathrm{p}} \mathrm{y}^{\mathrm{q}} \mathrm{f}(\mathrm{x}, \mathrm{y}) \mathrm{dx} \mathrm{dy}, p, q=0,1,2 \ldots
$$

Where, $\mathrm{D}$ is the area of each separated independent area, $x^{p} y^{q}$ as the transform kernel, $M_{p q}$ is called order geometric moments.

Here only the center of each target is required, therefore, $M_{00}, M_{01}, M_{10}$ are necessary. The center (x, y) of each object will be calculated as following:

$$
x=\frac{M_{10}}{M_{00}}, y=\frac{M_{01}}{M_{00}}
$$

\section{Extraction on Multiple Targets and Positioning}

In order to verify the above developed algorithms, the following experiments are carried.

The first experiment is done as shown in figure 4 . We take a picture with black circle marks. After the target areas are separated from non-target areas, the target areas are located with the moment invariant algorithms. Just like the results shown in figure 4-2, the center of each black circle is marked with a red dot. The result shows the multiple targets can be extracted accurately in one captured image with our developed image processing algorithms.

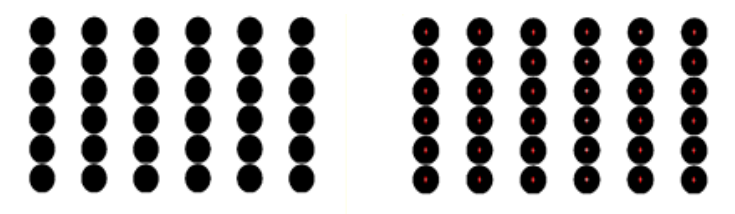

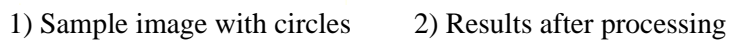
Fig.4 Extraction on multiple targets and center positioning

The second experiment is done in our lab with the image just like the picture shown in figure 3. In that image, there are three marks: two purple circle marks and one green circle mark. It can be easily found the influence of the lighting in figure 3 .

Based on the steps explained in section 2, the purple color mark modal and green mark modal are built. With the color modals, the target areas are separated from non-target areas. In figure 5, the targets can be extracted effectively even under the influence of lighting. Then using the moment invariants, the center of each mark is located.
1)Two purple marks

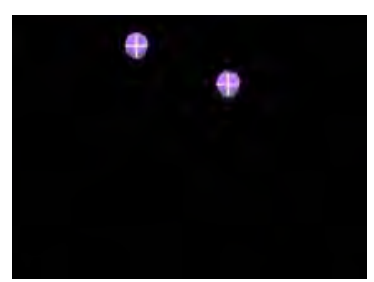

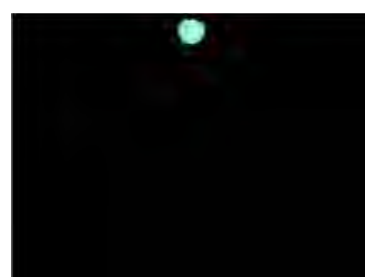

2) One green mark
Fig.5 Positioning multiple marks in lab lighting environments

Figure 6 shows the arm robot move to one target based on the position obtained with image processing. 
J. Wang, X. Zhang, W. Zhang and M. Sugisaka

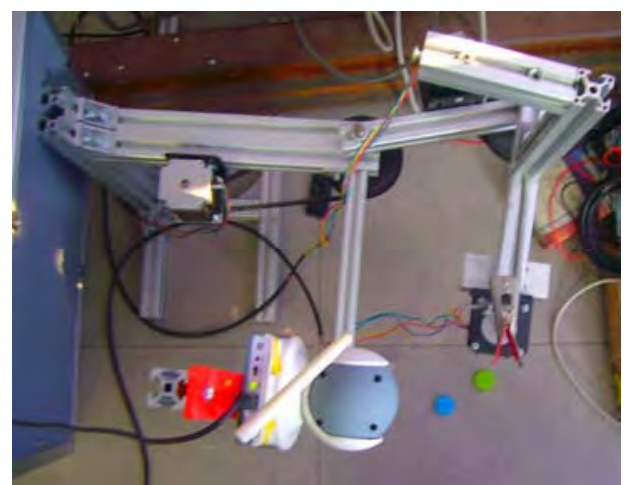

Fig. 6 Illustration on the arm robot picking one target

\section{Conclusions}

In this paper, an arm robot and the position control system based on CCD camera is developed. By fusion of color based target detection and moment invariants, the multiple targets can be extracted efficiently and their positions can be calculated. Because the multiple targets can be obtained in one captured image, unlike previous arm robot that can only move based on each input signal, the robot can be operated automatically to move to each target based on their different locations.

\section{References}

1. Y. B. Wang, Y. Z. Hu, and M. Y. Lu, Siemens machine vision system and its application in automobile engine assembly line, Automation systems engineering , 1 (2006) 20-22.

2. K. L. Zhuang, J. Z. Wang, and J. Zhou, Application of machine vision in angle inspection, Equipment Manufacturing Technology, 4(2011) 4-10.

3. G. L. David, Distinctive image features from scaleinvariant key points. Int. Journal of Computer Vision, 2(60)(2004) 91-110.

4. A. Khotanzad, Y. Huahong, Rotation invariant image recognition using features selected via a systematic method, Pattern recognition, 23(10)(1990) 1089-1101.

5. M. Sugisaka, X. Wang, Intelligent control of a mobile vehicle using on-line learning, System science, 25(1999) 41-50.

6. M. Sugisaka, X. Wang, J. Lee, Intelligent control strategy for a mobile vehicle, Applied mathematics and computation, 20(1998) 91-98. 